procedures. Transthoratic echocardiographic (TTE) and left ventricular angiography were performed before and after occluder deployment to confirm no residual shunt and aortic valve regurgitation. All patients were followed up at 1, 3, 6, and 12 months after the procedure and once a year thereafter. Clinical examinations, electrocardiagraphy (ECG), and TTE were conducted during the follow up visits.

Results From December 2001 to December 2010, 234 patients had been deployed short-waist occluders $(1.8 \mathrm{~mm})$ and 571 had longwaist occluders (3.5 mm). 98.3\% (805/819) of the patients, $98.7 \%$ in the short-waist group and $97.9 \%$ in the long-waist group, had successful closure of VSD during the procedure. Double-disc occluders were used in both treatment groups. No baseline characteristics in two groups, such as age, gender, body weight affect the treatment results. Average follow-up period was 35 months (34.2 \pm 21.9 ). Patients treated with long-waist occluders had significantly less arrhythmias, including complete right bundle branch block (CRBBB), complete left bundle branch block (CLBBB), incomplete right bundle branch block (IRBBB), left anterior bundle branch block (LABBB), junctional tachycardia, or complete atrial ventricular block (cAVB) than that in the short-waist group $(p=0.001)$. Particularly, 7 (3.0\%) patients in the short-waist group had cAVB and $3(1.3 \%)$ required permanent pace-maker insertion. In the longwaist group, $4(0.7 \%)$ patients had cAVB and $1(0.18 \%)$ had permanent pace-maker placement. The difference was statistically significant $(p=0.017)$. Other complications in addition to postoperative arrhythmias were also monitored after the procedure. In short-waist group, incidence of other complications, such as haemolysis, groin haematoma, residual shunt and valve regurgitation, was $31.6 \%$ (74 patients). In the long-waist group, the incidence was numerically lower. 140 patients $(24.5 \%)$ had complications mentioned above. The differences between two groups are not statistically significant $(p=0.091)$. After in-hospital medical management, groin haematoma and hemalysis were disappeared in all patients. In 1-year follow all residual shunt were recovered. 21 $(9.0 \%)$ patients in short-waist group had minor or minimal valve regurgitation, while in long-waist group, 64 (11.2\%) patients had such complications. No statistical significant differences were noted between two groups $(p=0.379)$.

Conclusions Waist-length of VSDOs might be related to post-procedural severe AVB in congenital VSD patients underwent transcatheter occlusion.

\section{Congenital heart disease and interventions GW23-e1025 TRANSCATHETER CLOSURE OF PERIMEMBRANOUS VENTRICULAR SEPTAL DEFECTS USING DIFFERENT OCCLUDERS: WAIST-LENGTH AND POST-PROCEDURAL ARRHYTHMIAS}

doi:10.1136/heartjnl-2012-302920p.1

Wu Hong, Wu Hong. Department of Cardiology, Changhai Hospital

Objectives Transcatheter closure of ventricular septal defects (VSD) has become a promising treatment alternative to surgery in the past decade. However, excessive risk of post-procedural arrhythmias pose a major challenge for broad utilising of this technology. In order to explore the mechanism associated with post-procedural arrhythmias and decrease the risk, we evaluated the relationship between different waist-length occluders and incidence of postprocedural arrhythmias.

Methods In this retrospective study, 819 VSD patients who had been treated with transcatheter occluders in our centre from December 2001 to December 2010 were evaluated. Patients were treated in two groups, short-waist group and long-waist group, based on different waist-sized occluders used in the transcatheter 